\title{
Análise da relação do quartil de nascimento com o estágio maturacional de jovens atletas
}

\author{
Analysis of the relationship between the birth quartile and the maturation stage of young athletes \\ Análisis de la relación entre el cuartil de nacimiento y la etapa de maduración de deportistas jóvenes
}

Recebido: 10/03/2021 | Revisado: 18/03/2021 | Aceito: 25/03/2021 | Publicado: 02/04/2021

\author{
Cláudio Hélio Lobato \\ ORCID: https://orcid.org/0000-0001-5848-566X \\ Universidade Federal do Rio Grande do Norte, Brasil \\ E-mail: chlobato@ufrn.edu.br \\ Paulo Francisco de Almeida-Neto \\ ORCID: https://orcid.org/0000-0002-2860-2260 \\ Universidade Federal do Rio Grande do Norte, Brasil \\ E-mail: paulojitte@ufrn.edu.br \\ Tatianny de Macêdo Cesário \\ ORCID: https://orcid.org/0000-0002-7813-1559 \\ Universidade Federal do Rio Grande do Norte, Brasil \\ E-mail: tatiannymc@gmail.com \\ Rui Barboza Neto \\ ORCID: https://orcid.org/0000-0002-5125-6773 \\ Universidade Federal do Rio Grande do Norte, Brasil \\ E-mail: tricolorrui@yahoo.com.br \\ Pablo Henrique Moura de Paiva \\ ORCID: https://orcid.org/0000-0003-4173-2411 \\ Universidade Federal do Rio Grande do Norte, Brasil \\ E-mail: pablo96529897@ hotmail.com \\ Rafaela Catherine da Silva Cunha de Medeiros \\ ORCID: https://orcid.org/0000-0003-2150-5190 \\ Universidade Federal do Rio Grande do Norte, Brasil \\ E-mail: rafaelacath@ hotmail.com \\ Paulo Moreira Silva Dantas \\ ORCID: https://orcid.org/0000-0002-9217-7107 \\ Universidade Federal do Rio Grande do Norte, Brasil \\ E-mail:pgdantas@icloud.com \\ Breno Guilherme de Araújo Tinoco Cabral \\ ORCID: https://orcid.org/0000-0002-9966-9956 \\ Universidade Federal do Rio Grande do Norte, Brasil \\ E-mail: brenotcabral@gmail.com
}

\begin{abstract}
Resumo
Muitos equívocos são cometidos diante da dificuldade em utilizar ferramentas na busca de identificação e seleção de talentos no esporte. Este estudo analisou a relação do quartil de nascimento com o estágio maturacional de jovens atletas. Foram selecionados 238 indivíduos, praticantes de esportes há pelo menos um ano, de ambos os sexos $(12,4 \pm 2,16$ anos). As datas de nascimento foram distribuídas de acordo com os quartis de nascimento correspondentes. A avaliação da maturação foi realizada a partir da subtração da idade óssea em relação a idade cronológica, a idade óssea foi mensurada por um modelo matemático de alta confiabilidade que utiliza variáveis antropométricas. Os quartis de nascimento não se associaram com o estágio de maturação biológica (masculino: $r=0.03, p=0.6$; feminino: $r=0,04$, $\mathrm{p}=0,6$ ). Os quartis de nascimento não foram significativos para prever o estágio de maturação biológica (masculino: $\beta=0,15, p=0,1$; feminino: $\beta=0,8, p=0,1)$. Em ambos os sexos, ao considerar os quartis de nascimento, os estágios maturacionais não apontaram diferenças significantes $(\mathrm{p}=0,2)$ e os sujeitos nascidos no mesmo ano e quartil apresentaram níveis de maturação biológica diferentes $\left(\eta^{2} \mathrm{p}<0,22\right)$. Tais resultados sugerem que não existe correlação entre os quartis de nascimento e os estágios maturacionais.
\end{abstract}

Palavras-chave: Desempenho esportivo; Esporte; Puberdade; Crianças; Adolescentes.

\begin{abstract}
Many mistakes are made due to the difficulty in using tools in the search for identification and selection of talents in the sport. This study analyzed the relationship between the birth quartile and the maturation stage of young athletes. A total of 238 individuals were selected, having practiced sports for at least one year, of both sexes (12.4 \pm 2.16 years). The birth dates were distributed according to the corresponding birth quartiles. The evaluation of maturation was performed by subtracting bone age from chronological age, bone age was measured by a highly reliable mathematical
\end{abstract}


model that uses anthropometric variables. The birth quartiles were not associated with the biological maturation stage (male: $\mathrm{r}=0.03, \mathrm{p}=0.6$; female: $\mathrm{r}=0.04, \mathrm{p}=0.6$ ). The birth quartiles were not significant for predicting the biological maturation stage (male: $\beta=0.15, p=0.1$; female: $\beta=0.8, p=0.1$ ). In both sexes, when considering the birth quartiles, the maturation stages did not show significant differences $(p=0.2)$ and the subjects born in the same year and quartile presented different levels of biological maturation $\left(\eta^{2} p<0.22\right)$. Such results suggest that there is no correlation between birth quartiles and maturation stages.

Keywords: Sports performance; Sport; Puberty; Children; Teenagers.

\section{Resumen}

Se cometen muchos errores debido a la dificultad de utilizar herramientas en la búsqueda de identificación y selección de talentos en el deporte. Este estudio analizó la relación entre el cuartil de nacimiento y la etapa de maduración de atletas jóvenes. Se seleccionaron un total de 238 individuos, con al menos un año de práctica deportiva, de ambos sexos $(12,4 \pm 2,16$ años). Las fechas de nacimiento se distribuyeron según los cuartiles de nacimiento correspondientes. La evaluación de la maduración se realizó restando la edad ósea de la edad cronológica, la edad ósea se midió mediante un modelo matemático altamente confiable que utiliza variables antropométricas. Los cuartiles de nacimiento no se asociaron con la etapa de maduración biológica (hombre: $r=0,03, p=0,6$; mujer: $r=0,04, p=0,6$ ). Los cuartiles de nacimiento no fueron significativos para predecir la etapa de maduración biológica (masculino: $\beta=0,15, \mathrm{p}=0,1$; femenino: $\beta=0,8, p=0.1$ ). En ambos sexos, al considerar los cuartiles de nacimiento, las etapas de maduración no mostraron diferencias significativas $(\mathrm{p}=0,2)$ y los sujetos nacidos en el mismo año y cuartil presentaron diferentes niveles de maduración biológica $\left(\eta^{2} p<0,22\right)$. Estos resultados sugieren que no existe correlación entre los cuartiles de nacimiento y las etapas de maduración.

Palabras clave: Rendimiento deportivo; Deporte; Pubertad; Niños; Adolescentes.

\section{Introdução}

Diversos são os instrumentos empregados a fim de identificar novos talentos no esporte (Patel et al., 2020). Dentre eles está a idade relativa, a qual pode ser representada pelo quartil (i.e., trimestre) de nascimento, e a idade biológica cujo referencial pode ser o estágio maturacional de puberdade (Patel et al., 2020).

Tais instrumentos se relacionam com a possibilidade de predizerem a antropometria e o desempenho físico (Figueiredo, 2020). Mas a maturação biológica nem sempre está de acordo com a idade cronológica, por isso os sujeitos podem ser classificados em atrasados, sincronizados e acelerados (Malina \& Bouchard, 2002).

Cotidianamente, profissionais envolvidos com o esporte na busca por novos talentos se encontram, indubitavelmente, passíveis de reflexões éticas no que tange o emprego da subjetividade e/ou utilização inadequada de instrumentos de seleção (Till \& Baker, 2020).

Entretanto, as chances de reconhecimento, aliado ao retorno financeiro que as vitórias em competições esportivas promovem, desencadeiam um maior aporte de recursos na seleção de talentos a fim de perenizar tais ganhos (Till \& Baker, 2020). Diante disso, se pode perceber dificuldades ou equívocos na utilização de ferramentas para o acompanhamento do atleta no esporte.

É admissível identificar atraso maturacional num atleta nascido no início do ano competitivo, bem como constatar estágio adiantado de maturação, mesmo com a idade relativa atrasada em relação ao início da competição (Megan et al., 2019).

Apesar do aumento significativo da utilização devido aos recursos limitados, nos últimos 15 a 20 anos, do Sistema de Identificação e Desenvolvimento de Talentos (TIDS, Cobley \& Till, 2015; Rongen et al., 2018), tanto organizações esportivas como praticantes, reconhecem os efeitos negativos da seleção precoce de atletas. Till \& Baker (2020) denotam a importância da responsabilidade dos profissionais na seleção de talentos para a saúde dos atletas e enfocam como o desencorajamento de aparente especialização precoce pode ser colocada em prática.

Johnston et al. (2017) aponta o fato de diversos estudos sobre o tema proposto enfatizarem características antropométricas e físicas de atletas em detrimento a investigação de fatores cognitivos e psicológicos. Além disso, se faz 
necessário analisar amostras de distintas localidades, a fim de verificar se há mudança no comportamento dos dados de acordo com o ambiente, condição socioeconômica, além de outras especificidades.

Atualmente, um dos grandes trunfos da ciências do esporte tem sido a busca por processos de otimização da seleção e orientação de talentos no esporte (Malina et al., 2015; Almeida-Neto et al., 2020, Almeida-Neto, Silva Dantas et al., 2020). Nos esportes de competição muitas questões estão associadas ao quartil de nascimento e estágio maturacional em que o indivíduo praticante de esporte se encontra, associados ao seu rendimento (Malina \& Bouchard, 2002; Malina et al., 2015). Dessa forma, o estudo em questão tem por objetivo investigar a associação do quartil de nascimento com o estágio maturacional.

\section{Métodos}

\section{Assuntos}

O presente estudo foi do tipo transversal e de acordo com Matsudo, Rivet \& Pereira (1987), a amostra se classifica como atletas de nível III (em escala crescente de I a VI) caracterizados por: (i) Realizarem educação física escolar. (ii) Fazerem parte de programa esportivo. (iii) Participarem de competições dentro de programa esportivo. O tamanho da amostra foi determinado com base em estudos anteriores (Sounis, 1971), considerando a variável maturação biológica (Almeida-Neto et al., 2020, Almeida-Neto, Silva Dantas et al., 2020). Deste modo, foi estimado um effect size de 0.80, um $\alpha<0.05$ e um $\beta=0.80$.

Utilizamos como critério de inclusão ser praticante de esportes há pelo menos um ano e ter nascido entre os anos de 2005 e 2011. Foram excluídos da pesquisa indivíduos que apresentaram alguma doença que os impediram de realizar as avaliações propostas pelo estudo e aqueles que não aceitaram participar da pesquisa.

\section{Ética}

Os indivíduos e seus responsáveis foram informados sobre os propósitos da pesquisa e os procedimentos metodológicos adotados no estudo, deste modo todos assinaram os termos de consentimento e assentimento livre e esclarescido. A pesquisa foi analisada e aprovada pelo Comitê de Ética e Pesquisa da Universidade Federal do Rio Grande do Norte (Parecer: 3.726.772), respeitando rigorosamente os princípios éticos contidos na declaração de Helsinki. E o estudo seguiu todas as recomendações do checklist STROBE para estudos observacionais (Von Elm et al., 2014).

\section{Antropometria}

As avaliações antropométricas foram baseadas no protocolo ISAK (International Society of the Advancement of Kinanthropometry) (Karupaiah, 2018). A massa corporal foi mensurada por meio da balança digital com variação de $0.10 \mathrm{~kg}$ (FILIZOLA $^{\circledR}$, Brasil). A estatura foi avaliada por um estadiômetro com precisão de $0.01 \mathrm{~cm}$ (SANNY ${ }^{\circledR}$, Brasil). As dobras cutâneas foram mensuradas com um adipômetro científico (SANNY ${ }^{\circledR}$, Brasil). A perimetria foi aferida com uma fita antropométrica (SANNY ${ }^{\circledR}$, Brasil) e para os diâmetros ósseos foi utilizado um paquímetro (SANNY ${ }^{\circledR}$, Brasil). As medidas antropométricas foram aferidas em três vezes consecutivas por um único avaliador e a mediana dos dados foram utilizadas para fins de análises.

\section{Avaliação da maturação}

A maturação foi aferida pela subtração da idade óssea em relação a idade cronológica (Malina \& Bouchard, 2002). Neste sentido, a idade óssea foi mensurada pela equação de Cabral et al., (2016), o modelo preditor consiste na fórmula: 


$$
\begin{gathered}
\text { Idade Óssea }=-11.620+7.004 *(\text { Estatura })+1.226 *(\text { Dsexo })+0.749 * \\
(\text { Idade })-0.068 *(\text { Dobra cutânea Tricipital })+0.214 *(\text { Circunferência do bíceps } \\
\text { braquial corrigido })-0.588 * \text { (Diâmetro ósseo do Úmero })+0.388 * \text { (Diâmetro ósseo } \\
\text { do fêmur }) .
\end{gathered}
$$

Legenda: Dsexo = utilizar valor 0 para o sexo masculino e valor 1 para o sexo feminino.

Deste modo, ao fim da equação a amostra foi classificada em maturação atrasada (resultados <-1), sincronizada (resultados entre -1 e 1) e acelerada (resultados > 1) (Malina \& Bouchard, 2002).

\section{Análise dos quartis}

Os sujeitos também foram classificados de acordo com os quartis do ano cronológico conforme a descrição:

Quadro 1. Classificação dos meses do ano de acordo com os quartis anuais.

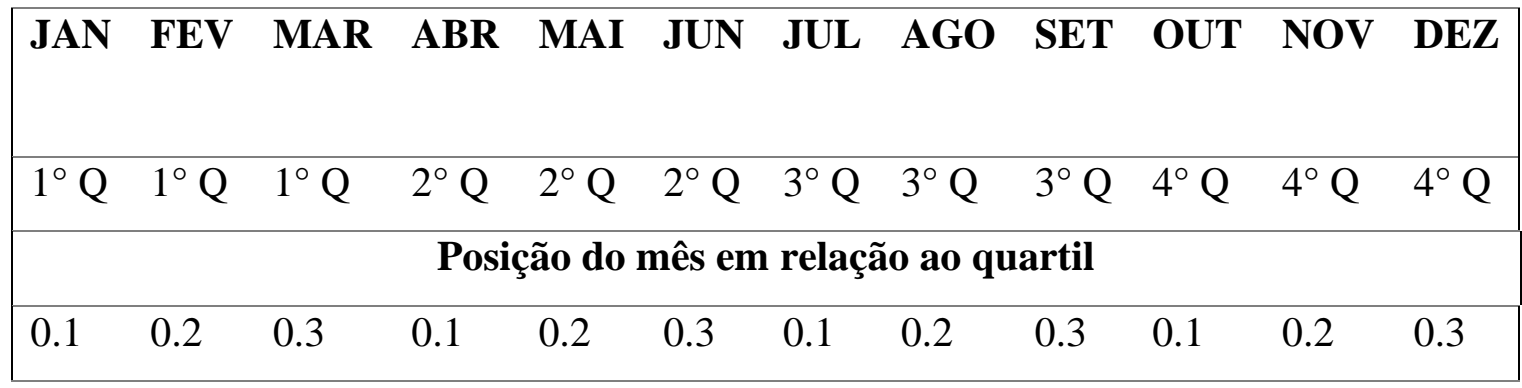

Legenda: Q = Quartil; JAN = Janeiro; FEV = Fevereiro; MAR = Março; ABR = Abril; MAI = Maio; JUN = Junho; JUL = Julho; AGO = Agosto; SET $=$ Setembro; OUT $=$ Outubro; NOV = Novembro; DEZ = Dezembro. Fonte: Autores (2021).

Para as análises estatísticas inserimos o valor do número equivalente a posição do mês de nascimento em relação ao quartil (Quadro 1) após o número do quartil, conforme o exemplo:

\section{Posição do mês de fevereiro dentro do quartil = 0.2}

Quartil do mês de fevereiro $=1$

\section{Classificação do indivíduo em relação ao quartil de nascimento= 1.2}

\section{Análises Estatísticas}

A normalidade foi verificada pelos testes de Kolmogorov-Smirnov e Z-score de assimetria e curtose (-1.96 a 1.96). As correlações dos dados foram feitas pelo teste de Pearson. A magnitude usada foi a de Schober, Boer \& Schwarte (2018): Insignificante: $\mathrm{r}<0.10$; Fraco: $\mathrm{r}=0.10-0.39$; moderado: $\mathrm{r}=0.40-0.69$; Forte: $\mathrm{r}=0.70-0.89$; Muito forte: $\mathrm{r}=0.90-1.00$. Posteriormente foi realizada uma análise de regressão linear, a homogeneidade foi medida pelo teste de Breush-Pegan e os pressupostos de normalidade, variância e independência dos dados não foram negados. A correção de Bonferroni foi aplicada e, posteriormente, foram realizadas comparações. As comparações entre os grupos [masculino x feminino] foram feitas pelo teste T independente de Student. As comparações por análise da variância entre os grupos [quartil 1 x quartil 2 x quartil 3 x quartil 4] foram realizada utilizando-se o teste ANOVA One-Way, em seguida o post-hoc de Bonferroni, foi aplicado para identificar as diferenças pontuais. O Eta-quadrado parcial $\left(\eta^{2} p\right)$ foi utilizado para verificar o tamanho do efeito da interação entre as variâncias das variáveis: [maturação $\times$ quartis do ano]; [sexo $\times$ maturação]; [sexo $\times$ quartis do ano]. A magnitude de $\eta^{2} p$ foi interpretada da seguinte forma: Pequeno $\eta^{2} p \leq 0,10$ a 0,$23 ; \eta^{2} p$ médio de 0,24 a 0,34 ; Grande $\eta^{2} p$ de 0,35 a 0,44 ; Muito grande $\eta^{2} p \geq 0,45$ 
(Cohen, 1988). O erro técnico das medidas antropométricas foi analisado da seguinte forma: Aceitável $\leq 1.0 \%$ (Perini et al., 2005). Todas as análises foram feitas no software open Source R (versão 4.0.1) considerando a significância de $\mathrm{p}<0.05$.

\section{Resultados}

A amostra foi composta por 238 indivíduos de ambos os sexos residentes da cidade de Natal / RN - Brasil (sendo 54.4 $\%$ do sexo masculino e $45.5 \%$ do sexo feminino, com média de idade de $12.4 \pm 2.16$ anos).

A Tabela 1 informa a caracterização da amostra. Os indivíduos apresentaram média de idade óssea e de maturação classificadas como sincronizada em relação a idade cronológica e nas comparações entre as características dos indivíduos do sexo masculino e feminino não houve diferenças estatísticas significantes ( $\mathrm{p}>0.05$ ). Além disso, para todas as medidas antropométricas foram indicados erros de medidas $<1 \%$. Em adição, destacamos que não houve perdas amostrais e que a margem de possibilidade de erro apontada para o tamanho da amostra foi de $4.97 \%$, estando abaixo de 5\% (dentro da margem de confiança de $95 \%$ ) e indicando um poder amostral de 0.80, sugerindo que a amostra possui força estatística para responder a pergunta de pesquisa.

Tabela 1. Caracterização da amostra quanto a mensuração corporal e ao quartil de nascimento.

\begin{tabular}{lcccc}
\hline Variáveis & Toda amostra & Masculino & Feminino & $\mathrm{p}$ \\
\hline $\mathrm{n}^{\circ}(\%)$ & $238(100 \%)$ & $130(54.5 \%)$ & $108(45.5 \%)$ & ------ \\
Idade (Anos) & $12.4 \pm 2.16$ & $12.4 \pm 3.24$ & $12.4 \pm 2.16$ & 0.2 \\
Idade óssea & $12.5 \pm 4.14$ & $12.3 \pm 4.13$ & $12.5 \pm 3.69$ & 0.3 \\
Maturação (Idade óssea) & $0.06 \pm 3.69$ & $0.06 \pm 4.13$ & $0.07 \pm 3.69$ & 0.1 \\
Estatura $(\mathrm{Cm})$ & $154.0 \pm 0.14$ & $154.2 \pm 0.40$ & $154.1 \pm 13.8$ & 0.9 \\
Massa corporal $_{(\mathrm{Kg})}$ & $46.4 \pm 13.9$ & $46.5 \pm 13.9$ & $46.4 \pm 13.9$ & 0.08 \\
${\text { Índice de massa corporal }\left(\mathrm{m}^{2}\right)}$ & $19.1 \pm 3.57$ & $19.0 \pm 3.55$ & $19.1 \pm 3.57$ & 0.6 \\
$1^{\circ}$ quartil $(\mathrm{n} \%)$ & $78(33 \%)$ & $43(33 \%)$ & $35(33 \%)$ & ------ \\
$2^{\circ}$ quartil $(\mathrm{n} \%)$ & $53(22 \%)$ & $30(23 \% \%)$ & $23(21 \% \%)$ & ------ \\
$3^{\circ}$ quartil $(\mathrm{n} \%)$ & $59(25 \%)$ & $34(26 \%)$ & $25(23 \%)$ & ------ \\
$4^{\circ}$ quartil $(\mathrm{n} \%)$ & $48(20 \%)$ & $23(18 \%)$ & $25(23 \%)$ & ------ \\
\hline
\end{tabular}

Legenda: $\mathrm{n}=$ número absoluto. $\%=$ Percentual. $(\mathrm{Cm})=$ centímetros; $(\mathrm{Kg})=$ Quilogramas; $\left(\mathrm{m}^{2}\right)=$ Metro quadrado. Fonte: Autores $(2021)$.

Destaca-se que nas análises de correlação os quartis de nascimento do ano não se associaram de forma significante com a maturação biológica (amostra total: $r=0.03$; $p=0.9$; masculino: $r=0.03$; $p=0.6$; feminino: $r=0.04$; $p=0.6$ ). Ademais, se pode verificar na Tabela 2 que os quartis de nascimento do ano não apresentam significância estatística (p>0.05) para indicar a previsão da maturação, peso ou estatura corporal. 
Tabela 2. Análises de regressão linear das variáveis maturação, peso e estatura corporal com os quartis do ano cronológico.

\begin{tabular}{|c|c|c|c|c|c|c|c|c|c|c|c|c|}
\hline & \multicolumn{3}{|c|}{ Toda amostra } & \multicolumn{4}{|c|}{ Masculino } & \multicolumn{5}{|c|}{ Feminino } \\
\hline & $\mathrm{r}^{2}$ & $\beta$ & $F_{(236)}$ & $\mathrm{p}$ & $\mathrm{r}^{2}$ & $\beta$ & $F_{(116)}$ & $\mathrm{p}$ & $\mathrm{r}^{2}$ & $\beta$ & $F_{(106)}$ & $\mathrm{p}$ \\
\hline Maturação & 0.00 & 0.11 & 0.27 & 0.1 & 0.00 & 0.15 & 0.17 & 0.1 & 0.00 & 0.08 & 0.17 & 0.1 \\
\hline Peso $(\mathrm{Kg})$ & 0.00 & 0.02 & 0.00 & 0.1 & 0.00 & 0.23 & 0.05 & 0.1 & 0.00 & -0.19 & 0.03 & 0.1 \\
\hline Estatura $(\mathrm{Cm})$ & 0.00 & -0.04 & 0.39 & 0.1 & 0.00 & 0.06 & 0.00 & 0.1 & 0.00 & -0.81 & 0.64 & 0.1 \\
\hline
\end{tabular}

Legenda: $\mathrm{r}^{2}$ = coeficiente de determinação da regressão; $\beta=$ coeficiente de regressão angular em relação à variável dependente; $\mathrm{F}=$ Valor da significância global da regressão. Fonte: Autores (2021).

Ao segregarmos a amostra pelos quartis do ano foi verificado que, em ambos os sexos, o estágio de maturação biológica não apontou diferença significante entre os quartis do ano (todos apontaram maturação com classificação sincronizada) (p=0.2). Em adição, a maturação biológica ( $\eta^{2} \mathrm{p}<0.22$ para ambos os sexos) e o sexo ( $\eta^{2} \mathrm{p}<0.10$ para ambos os sexos) não apontaram interações significativas durante as comparações.

Figura 1. Comparações entre os estágios maturacionais dividindo a amostra por quartis.

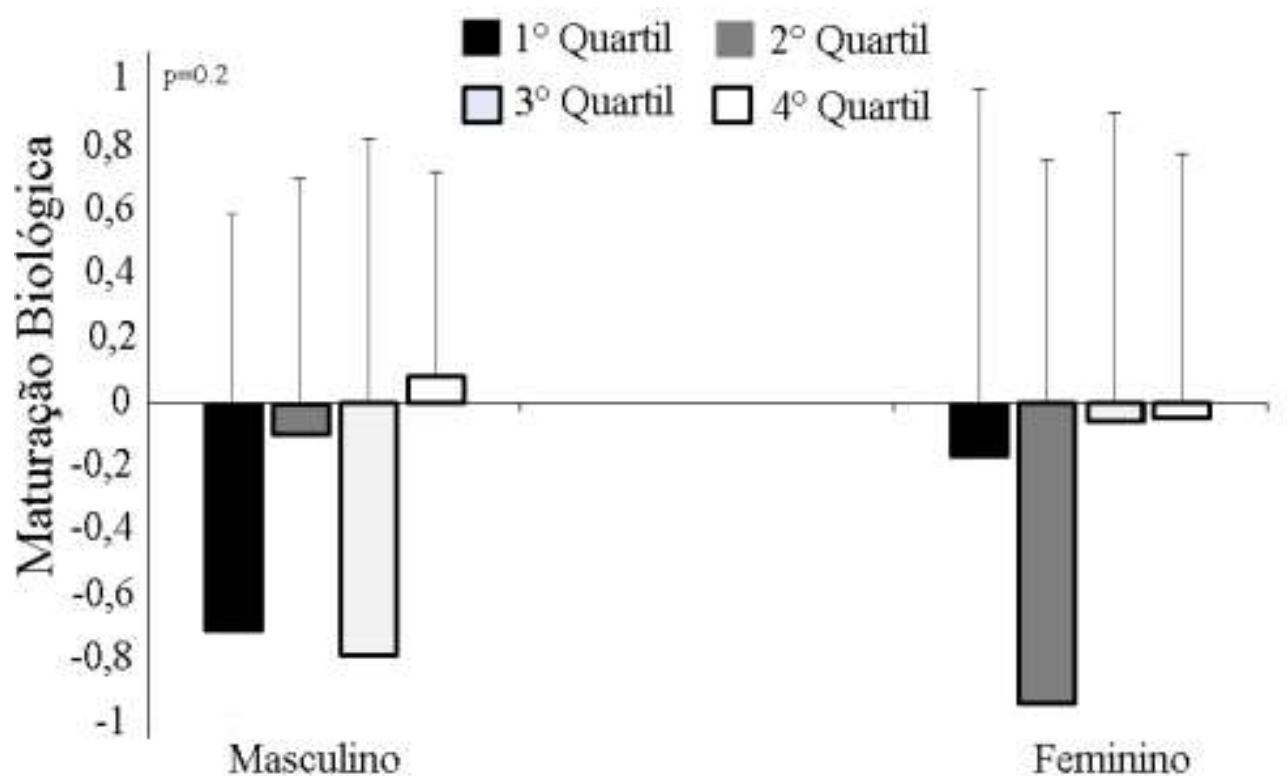

Fonte: Autores (2021).

A Tabela 3 retrata de forma descritiva que os sujeitos nascidos no mesmo ano e no mesmo quartil apresentam níveis de classificação maturacional diferentes independente do sexo. 
Tabela 3. Tabela descritiva da amostra em relação ao ano de nascimento, estágio maturacional e quartil do ano.

\begin{tabular}{|c|c|c|c|c|c|c|c|}
\hline \multirow[t]{2}{*}{ Ano } & \multirow[t]{2}{*}{ Nível Maturação } & \multicolumn{2}{|c|}{ Sexo } & \multicolumn{4}{|c|}{ Quartil } \\
\hline & & $\mathbf{F}$ & $\mathbf{M}$ & $\mathbf{1}^{\circ}$ & $2^{\circ}$ & $3^{\circ}$ & $4^{\circ}$ \\
\hline & Sincronizada & 11 & 21 & $3 \mathrm{~F} / 9 \mathrm{M}$ & $2 \mathrm{~F} / 5 \mathrm{M}$ & $3 \mathrm{~F} / 5 \mathrm{M}$ & $3 \mathrm{~F} / 2 \mathrm{M}$ \\
\hline \multirow[t]{3}{*}{2005} & Atrasada & 6 & 8 & $2 \mathrm{~F} / 2 \mathrm{M}$ & $1 \mathrm{~F} / 2 \mathrm{M}$ & $1 \mathrm{~F} / 2 \mathrm{M}$ & $2 \mathrm{~F} / 2 \mathrm{M}$ \\
\hline & Acelerada & 10 & 9 & $3 \mathrm{~F} / 2 \mathrm{M}$ & $2 \mathrm{~F} / 2 \mathrm{M}$ & $2 \mathrm{~F} / 3 \mathrm{M}$ & $3 \mathrm{~F} / 2 \mathrm{M}$ \\
\hline & Sincronizada & 2 & 11 & $0 \mathrm{~F} / 6 \mathrm{M}$ & $0 \mathrm{~F} / 2 \mathrm{M}$ & $2 \mathrm{~F} / 1 \mathrm{M}$ & $0 \mathrm{~F} / 2 \mathrm{M}$ \\
\hline \multirow[t]{3}{*}{2006} & Atrasada & 12 & 8 & $2 \mathrm{~F} / 2 \mathrm{M}$ & $4 \mathrm{~F} / 1 \mathrm{M}$ & $3 \mathrm{~F} / 1 \mathrm{M}$ & $3 \mathrm{~F} / 4 \mathrm{M}$ \\
\hline & Acelerada & 5 & 7 & $2 \mathrm{~F} / 0 \mathrm{M}$ & $0 \mathrm{~F} / 2 \mathrm{M}$ & $2 \mathrm{~F} / 4 \mathrm{M}$ & $1 \mathrm{~F} / 1 \mathrm{M}$ \\
\hline & Sincronizada & 3 & 13 & $1 \mathrm{~F} / 3 \mathrm{M}$ & $1 \mathrm{~F} / 5 \mathrm{M}$ & $1 \mathrm{~F} / 3 \mathrm{M}$ & $0 \mathrm{~F} / 2 \mathrm{M}$ \\
\hline \multirow[t]{3}{*}{2007} & Atrasada & 10 & 7 & $4 \mathrm{~F} / 3 \mathrm{M}$ & $3 \mathrm{~F} / 1 \mathrm{M}$ & $2 \mathrm{~F} / 2 \mathrm{M}$ & $1 \mathrm{~F} / 1 \mathrm{M}$ \\
\hline & Acelerada & 8 & 6 & $2 \mathrm{~F} / 0 \mathrm{M}$ & $3 \mathrm{~F} / 4 \mathrm{M}$ & $1 \mathrm{~F} / 1 \mathrm{M}$ & $2 \mathrm{~F} / 1 \mathrm{M}$ \\
\hline & Sincronizada & 7 & 5 & $2 \mathrm{~F} / 3 \mathrm{M}$ & $2 \mathrm{~F} / 0 \mathrm{M}$ & $1 \mathrm{~F} / 2 \mathrm{M}$ & $2 \mathrm{~F} / 0 \mathrm{M}$ \\
\hline \multirow[t]{3}{*}{2008} & Atrasada & 8 & 12 & $1 \mathrm{~F} / 4 \mathrm{M}$ & $1 \mathrm{~F} / 2 \mathrm{M}$ & $1 \mathrm{~F} / 4 \mathrm{M}$ & $5 \mathrm{~F} / 2 \mathrm{M}$ \\
\hline & Acelerada & 5 & 3 & $4 \mathrm{~F} / 1 \mathrm{M}$ & $0 \mathrm{~F} / 1 \mathrm{M}$ & $1 \mathrm{~F} / 0 \mathrm{M}$ & $0 \mathrm{~F} / 1 \mathrm{M}$ \\
\hline & Sincronizada & 6 & 1 & $4 \mathrm{~F} / 0 \mathrm{M}$ & $0 \mathrm{~F} / 0 \mathrm{M}$ & $1 \mathrm{~F} / 1 \mathrm{M}$ & $1 \mathrm{~F} / 0 \mathrm{M}$ \\
\hline \multirow[t]{3}{*}{2009} & Atrasada & 2 & 9 & $0 \mathrm{~F} / 4 \mathrm{M}$ & $1 \mathrm{~F} / 2 \mathrm{M}$ & $1 \mathrm{~F} / 1 \mathrm{M}$ & $0 \mathrm{~F} / 2 \mathrm{M}$ \\
\hline & Acelerada & 2 & 1 & $1 \mathrm{~F} / 0 \mathrm{M}$ & $1 \mathrm{~F} / 0 \mathrm{M}$ & $0 \mathrm{~F} / 1 \mathrm{M}$ & $0 \mathrm{~F} / 0 \mathrm{M}$ \\
\hline & Sincronizada & 2 & 0 & $1 \mathrm{~F} / 0 \mathrm{M}$ & $0 \mathrm{~F} / 0 \mathrm{M}$ & $1 \mathrm{~F} / 0 \mathrm{M}$ & $0 \mathrm{~F} / 0 \mathrm{M}$ \\
\hline \multirow[t]{3}{*}{2010} & Atrasada & 4 & 3 & $2 \mathrm{~F} / 1 \mathrm{M}$ & $1 \mathrm{~F} / 0 \mathrm{M}$ & $1 \mathrm{~F} / 1 \mathrm{M}$ & $0 \mathrm{~F} / 1 \mathrm{M}$ \\
\hline & Acelerada & 2 & 1 & $1 \mathrm{~F} / 0 \mathrm{M}$ & $0 \mathrm{~F} / 0 \mathrm{M}$ & $0 \mathrm{~F} / 1 \mathrm{M}$ & $1 \mathrm{~F} / 0 \mathrm{M}$ \\
\hline & Sincronizada & 0 & 1 & $1 \mathrm{~F} / 1 \mathrm{M}$ & $0 \mathrm{~F} / 0 \mathrm{M}$ & $1 \mathrm{~F} / 0 \mathrm{M}$ & $0 \mathrm{~F} / 0 \mathrm{M}$ \\
\hline \multirow[t]{2}{*}{2011} & Atrasada & 2 & 3 & $2 \mathrm{~F} / 2 \mathrm{M}$ & $1 \mathrm{~F} / 1 \mathrm{M}$ & $1 \mathrm{~F} / 0 \mathrm{M}$ & $0 \mathrm{~F} / 0 \mathrm{M}$ \\
\hline & Acelerada & 1 & 1 & $1 \mathrm{~F} / 0 \mathrm{M}$ & $0 \mathrm{~F} / 0 \mathrm{M}$ & $0 \mathrm{~F} / 1 \mathrm{M}$ & $1 \mathrm{~F} / 0 \mathrm{M}$ \\
\hline
\end{tabular}

Legenda: F = Feminino; $M$ = Masculino. Fonte: Autores (2021).

\section{Discussão}

O objetivo deste estudo foi investigar a associação do quartil de nascimento com o estágio maturacional de jovens atletas de ambos os sexos. Neste sentido, os principais resultados apontaram que os quartis de nascimento não se associaram com o estágio de maturação biológica, peso e estatura corporal. Além disso, Os quartis de nascimento não foram significativos para prever o estágio de maturação biológica, peso e estatura corporal. Em ambos os sexos, ao considerar os quartis de nascimento, os estágios maturacionais não apontaram diferenças significantes entre os quartis. Assim como, os sujeitos nascidos no mesmo ano e no mesmo quartil apresentaram níveis de maturação biológica diferentes.

De acordo com os resultados obtidos, o quartil de nascimento não está associado ao estágio maturacional. Tal achado sugere que jovens atletas nascidos em quartis diferentes podem competir na mesma categoria. Entretanto, o estágio maturacional precisa ser levado em consideração ao segregar as categorias esportivas (Malina \& Bouchard, 2002). Em estudos anteriores do 
nosso grupo, ao analisarmos jovens atletas de idade cronológica similar evidenciamos que, com o avançar da maturação biológica, os jovens atletas em estágio acelerado de maturação demonstram superioridade em relação ao desempenho neuromuscular de membros superiores e inferiores, o que fortalece a sugestão do presente estudo em relação a consideração da maturação biológica para a divisão de categorias esportivas (Almeida-Neto et al., 2020; Almeida-Neto, Silva Dantas et al., 2020; Almeida-Neto, de Matos et al., 2020; De Almeida-Neto et al., 2020).

Além disso, segundo Lloyd et al. (2014) e Malina et al. (2015) a maturação biológica também deve ser considerada durante o processo de seleção de jovens talentos esportivos, ao considerar a sua associação com o desempenho neuromuscular, a maturação pode ajudar a direcionar os treinamentos durante o processo de iniciação esportiva.

Em adição, Zhao et al. (2019) salientam, que a iniciação em determinados esportes requer um perfil cujo requisito não se aplica a outros, é necessário guiar a identificação e seleção de talentos de acordo com as especificidades de cada prática. No caso da ginástica, por exemplo, é desejável iniciar a prática em indivíduos que apresentem atraso maturacional, visto que a maturação tardia tem sido constatada em diversos atletas de sucesso (Malina, 1994). Till \& Baker (2020) destacam que além das características maturacionais, outros fatores a serem levados em consideração a fim de possibilitar a otimização do processo de identificação e seleção de talentos: histórico familiar, identificação com a modalidade, aspectos técnicos, táticos e psicológicos, habilidades específicas do esporte, dentre outros.

No presente estudo também foi identificado que o quartil de nascimento, além de não ser um preditor em potencial da maturação, não foi um preditor significante para variáveis morfológicas como peso e estatura corporal. Hill et al. (2019) sugerem que crianças de mesma idade com estatura mais próxima da que teriam na idade adulta são mais maturadas que aquelas mais distantes da altura adulta esperada (i.e., não havendo relação com o quartil de nascimento, mas com o estágio maturacional), o que corrobora com os achados no presente estudo, onde o quartil de nascimento não foi apontado estando associado à estatura corporal.

Destacamos que o presente estudo não encontrou diferenças significantes ao analisar os estágios maturacionais entre os quartis de nascimento. Este achado diverge dos de Penna et al. (2012), os quais encontraram relações significantes do quartil do ano com a estatura, maturação e com o desempenho de atletas sub-17 em campeonatos mundiais de futebol. Penna et al. (2012) sugerem vantagens quanto à variável maturacional "estatura" aos atletas nascidos no primeiro quartil frente aos demais. O estudo aponta que 41,5\% dos atletas presentes nas competições mundiais de 2007, 2009 e 2011 nasceram neste quartil. Expõe ainda diferenças significativas entre os nascidos no segundo quartil e os nascidos no terceiro e quarto quartis.

Desta forma, artigos publicados por nosso grupo de pesquisa vêm ratificar a ideia de que a maturação influencia a composição corporal, cognição e os níveis de força, tanto de membros inferiores quanto superiores, em diversas modalidades esportivas (Pinto et al., 2017; Dantas et al., 2018; Simões et al., 2018; Campos et al., 2019; Almeida-Neto et al., 2020; AlmeidaNeto, Silva Dantas et al., 2020; Almeida-Neto, de Matos et al., 2020; De Almeida-Neto et al., 2020; De Macêdo Cesário et al., 2020). Contudo, foi verificado que tais estudos não consideraram o quartil de nascimento.

Neste sentido, ao investigar tal aresta, o presente trabalho fortalece os resultados de estudos anteriores do nosso grupo em relação a consideração do uso da maturação biológica durante o processo de seleção de jovens talentos esportivos (Pinto et al., 2017; Simões et al., 2018; Campos et al., 2019; Almeida-Neto et al., 2020; De Almeida-Neto et al., 2020). Contudo, apesar dos achados relevantes, o presente estudo possui a limitação de ser do tipo observacional, impossibilitando estabelecer a relação causa e efeito, o que exige coerência ao interpretar os achados da presente pesquisa.

\section{Conclusão}

Os resultados do presente estudo permitem concluir que os quartis do ano não se correlacionam com os estágios de maturação biológica, assim como não predizem a maturação biológica em jovens atletas. Sugerindo que, isoladamente, o quartil 
de nascimento é incapaz de determinar o estágio maturacional de jovens atletas de ambos os sexos. Assim, se recomenda a produção de novos estudos a fim de refutar os dados apresentados.

\section{Agradecimentos}

Pelo apoio e incentivo para o desenvolvimento deste artigo acadêmico, agradecemos à Universidade Federal do Rio Grande do Norte (UFRN), à base de pesquisa Atividade Física e Saúde (AFISA), ao Grupo de Pesquisa em Maturação da Criança e do Adolescente (GEPMAC). Ao Conselho Nacional de Desenvolvimento Científico (CNPQ) e a Coordenação de Aperfeiçoamento de Pessoal de Nível Superior (CAPES).

\section{Referências}

Almeida-Neto, P. F.; de Matos, D. G.; Baxter-Jones, A. D. G.; Batista, G. R.; Pinto, V. C. M.; Dantas M.; Aidar, F. J.; Dantas P. M. S.; Cabral, B. G. A. T. (2020) The effectiveness of biological maturation and lean mass in relation to muscle strength performance in elite young athletes. Sustainability, 12 (17), 6696. $10.3390 /$ su 12176696

Almeida-Neto, P. F., Silva Dantas, P. M., Pinto, V. C. M., Cesário, T. D. M., Ribeiro Campos, N. M., Santana, E. E., \& Tinoco Cabral, B. G. D. A. (2020). Biological Maturation and Hormonal Markers, Relationship to Neuromotor Performance in Female Children. International Journal of Environmental Research and Public Health, 17(9), 3277.

Almeida-Neto, P. F. D., de Matos, D. G., Pinto, V. C. M., Dantas, P. M. S., Cesário, T. D. M., da Silva, L. F., \& Cabral, B. G. D. A. T. (2020). Can the Neuromuscular Performance of Young Athletes Be Influenced by Hormone Levels and Different Stages of Puberty? International Journal of Environmental Research and Public Health, 17 (16), 5637. 10.3390/ijerph17165637

Cabral, S. D. A. T., Cabral, B. G. D. A. T., Pinto, V. C. M., Andrade, R. D. D., Borges, M. V. D. O., \& Dantas, P. M. S. (2016). Relationship of age with bone and fitness anthropometry practitioners in youth volleyball. Revista Brasileira de Ciências do Esporte, 38(1), 69-75.

Campos, N. M., Santos, G. H., Teixeira, R. V., da Silva, L. M., de S Barbosa, P. R., Santana, E. E., \& Breno, G. D. A. (2019). Maturação e controle inibitório: Implicações para o processo de seleção de talentos. Motricidade, 15, 128-133.

Cohen, J. (1988). The t test for means. Statistical power analysis for the behavioural sciences. éd: Hillsdale, NJ: Lawrence Erlbaum Associates.

Dantas, M. P., Silva, L. F., Gantois, P., Silva, L. M., Dantas, R. N., \& Cabral, B. T. (2018). Relationship between maturation and explosive strength in young rowers/ Relação entre maturacao e forca explosiva em remadores jovens. Motricidade, 14(S1), 112-121.

De Almeida-Neto, P. F., Arrais, R. F., de Matos, D. G., Cesário, T. D., Correia, A. B., Silva, L. F., \& Cabral, B. G. (2020). The influence of biological maturation and neuromuscular performance on peak bone mineral density in children. The Journal of Sports Medicine and Physical Fitness.

De Macêdo Cesário, T., de Almeida-Neto, P. F., de Matos, D. G., Wells, J., Aidar, F. J., \& Cabral, B. G. A. T. (2020). Evaluation of the body adiposity index against dual-energy X-ray absorptiometry for assessing body composition in children and adolescents. American Journal of Human Biology, e23503.

Figueiredo, A. J., Coelho e Silva, M. J., \& Malina, R. M. (2011). Predictors of functional capacity and skill in youth soccer players. Scandinavian journal of medicine \& science in sports, 21(3), 446-454.

Johnston, K., Wattie, N., Schorer, J., \& Baker, J. (2018). Talent identification in sport: a systematic review. Sports Medicine, 48(1), 97-109.

Karupaiah, T. (2018). Limited (ISAK) profiling The International Society for the Advancement of Kinanthropometry (ISAK). Journal of Renal Nutrition and Metabolism, 3(1), 11-11.

Lloyd, R. S., Oliver, J. L., Faigenbaum, A. D., Myer, G. D., \& Croix, M. B. D. S. (2014). Chronological age vs. biological maturation: implications for exercise programming in youth. The Journal of Strength \& Conditioning Research, 28(5), 1454-1464. 10.1519/ JSC.0000000000000391

Malina, R. M., Rogol, A. D., Cumming, S. P., e Silva, M. J. C., \& Figueiredo, A. J. (2015). Biological maturation of youth athletes: assessment and implications. British Journal of Sports Medicine, 49(13), 852-859. 10.1136/bjsports-2015-094623

Malina, R. M., \& Bouchard, C. (2002). Atividade física do atleta jovem: do crescimento à maturação. Editora Roca.

Malina, R. M. (1994). Physical growth and biological maturation of young athletes. Exercise and sport sciences reviews, $22(1), 280-284$.

Matsudo, V. K., Rivet, R. E., \& Pereira, M. H. (1987). Standard score assessment on physique and performance of Brazilian athletes in a six tiered competitive sports model. Journal of sports sciences, 5(1), 49-53. Doi: 10.1080/02640418708729763

Megan, H., et al. (2019): Relative age and maturation selection biases in academy football, Journal of Sports Sciences. 10.1080/02640414.2019.1649524

Patel, R., Nevill, A., Smith, T., Cloak, R., \& Wyon, M. (2020). The influence of birth quartile, maturation, anthropometry and physical performances on player retention: observations from an elite football academy. International Journal of Sports Science \& Coaching, 15(2), 121-134. 10.1177 / 1747954120906507 
Research, Society and Development, v. 10, n. 4, e12710413740, 2021

(CC BY 4.0) | ISSN 2525-3409 | DOI: http://dx.doi.org/10.33448/rsd-v10i4.13740

Penna, E. M. (2012). Relação entre mês de nascimento e estatura de atletas do mundial de futebol sub 17. Revista brasileira cineantropometria desempenho humano, 571-581. 10.5007/1980-0037.2012v14n5p571

Perini, T. A. Lameira de Oliveira G., Santos Ornellas J., Palha de Oliveira F. (2005). Technicalerrorofmeasurement in anthropometry. Revista Brasileira de Medicina do Esporte, 11, 86-90.

Pinto, V. C. M., dos Santos, P. G. M. D., Dantas, M. P., Araújo, J. P. D. F., Cabral, S. D. A. T., \& Cabral, B. G. D. A. T. (2017). Relationship between skeletal age, hormonal markers and physical capacity in adolescents. Journal of Human Growth and Development, 27(1), 77-83. 10.7322/jhgd.127658

Schober, P., Boer, C., \& Schwarte, L. A. (2018). Correlation coefficients: appropriate use and interpretation. Anesthesia \& Analgesia, $126(5), 1763-1768$.

Simões, T. B., Santana, E. E., Dantas, I. C., Castro, K. R., Dantas, R. P., Araújo, J. P. F., \& Cabral, B. A. (2018). Marcadores cognitivos e prática esportiva em diferentes estágios maturacionais. Motricidade, 14.

Sounis, E. (1971). Bio estatística; princípios fundamentais, metodologia estatística. Aplicação às Ciências Biológicas. In Bio estatística; princípios fundamentais, metodologia estatística. Aplicação às Ciências Biológicas (pp. 228-228).

Till, K., \& Baker, J. (2020). Challenges and [possible] solutions to optimizing talent identification and development in sport. Frontiers in psychology, 11. 10.3389/fpsyg.2020.00664. PMID: 32351427;

Von Elm, E., Altman, D. G., Egger, M., Pocock, S. J., Gøtzsche, P. C., Vandenbroucke, J. P., \& Strobe Initiative. (2014). The Strengthening the Reporting of Observational Studies in Epidemiology (STROBE) Statement: guidelines for reporting observational studies. International journal of surgery, 12(12), 14951499 .

Zhao, K., Hohmann, A., Chang, Y., Zhang, B., Pion, J., \& Gao, B. (2019). Physiological, anthropometric, and motor characteristics of elite Chinese youth athletes from six different sports. Frontiers in physiology, 10, 405. 10.3389/fphys.2019.00405 